\title{
Comparação entre a incidência, nacional, estadual e do município de Vassouras, de sífilis congênita, nos últimos quinze anos
}

\author{
Comparison between the incidence, national, state and Vassouras municipality, of the congenital \\ syphilis, in the last fifteen years
}

Adriano Garcia Ferreira ${ }^{1,2^{*}}$, Victor Felipe Justiniano Barbosa ${ }^{1,2}$, Marise Maleck ${ }^{1,2,3,4,5}$

Como citar esse artigo. Ferreira

AG, Barbosa, VFJ, Maleck, M Comparação entre a incidência nacional, estadual e do município de Vassouras, de sífilis congênita, nos últimos quinze anos. Revista de Saúde. 2018 Jan./Jun.; 09 (1): 14-17.

\begin{abstract}
Resumo
A sífilis é uma doença crônica causada pela bactéria Treponema pallidum, geralmente adquirida por contato sexual com outro indivíduo infectado. As gestantes fazem parte de um importante grupo epidemiológico, uma vez infectadas pela bactéria T. pallidum, podem gerar crianças com sífilis congênita através da disseminação hematogênica transplacentária. Este estudo teve como objetivo realizar um levantamento epidemiológico da incidência de sífilis congênita no município de Vassouras, no estado do Rio de Janeiro e no Brasil. O levantamento foi realizado a partir dos informes epidemiológicos do município de Vassouras-RJ e no Brasil. Os resultados mostraram que o município de Vassouras revelou uma taxa de incidência de sífilis congênita compreendida entre 2 e 4,4 casos/1000 nascidos vivos, em 5 anos alternados e inferiores as observadas aos índices do Brasil e do Rio de Janeiro, apresentando inclusive incidência zero em dez anos dos quinze anos analisados. Devido a sua importância, este estudo epidemiológico poderá contribuir com a qualidade dos serviços de Atenção Básica à Saúde da Mulher no município de Vassouras, RJ.
\end{abstract}

Palavras-chave: Treponema pallidum; Ś́filis congênita; Saúde da Mulher.

\begin{abstract}
Syphilis is a chronic disease caused by the bacterium Treponema pallidum and is usually acquired through sexual contact with another infected individual. Pregnant women form part of an important epidemiological group, since once infected by the bacterium T. pallidum not only carry syphilis but can give birth to children with congenital syphilis through transplacental hematogenic dissemination. The aim of this study was to epidemiologically survey the incidence of congenital syphilis in the municipality of Vassouras, state of Rio de Janeiro, and throughout Brazil. The survey was conducted using epidemiological reports from Vassouras and the entire country, covering the last 15 years. The results showed that Vassouras had incidence rates for congenital syphilis ranging from 2 to 4.4 cases/1000 live births, in five of the 15 years analyzed and zero incidence in the other ten years. These rates were lower than those for the whole country and for the state of Rio de Janeiro. This epidemiological study provides important data that may contribute towards the quality of primary healthcare services for women in Vassouras, Rio de Janeiro.

Keywords: Treponema pallidum; Congenital syphilis; Women's health.
\end{abstract}

\section{Introdução}

A sífilis é uma doença crônica causada pela bactéria Treponema pallidum, geralmente adquirida por contato sexual com indivíduo infectado ${ }^{1,2}$. A sífilis apresenta uma ampla variedade de manifestações clínicas que, se não tratada, progride para os estágios primário, secundário, terciário da doença $a^{1,2}$. As gestantes fazem parte de um grupo epidemiológico importante, pois, quando infectadas pela bactéria causadora de sífilis, podem gerar crianças com sífilis congênita através da disseminação hematogênica transplacentária ${ }^{1,2}$. O teste "Venereal Disease Research Laboratory" (VDRL) deve ser realizado em todas as mulheres grávidas no início da gestação e deve ser repetido perto do término da gestação ${ }^{1}$. O risco de infecção fetal é maior nas fases iniciais da sífilis materna não tratada, uma vez que o não tratamento da gestante com sífilis pode resultar em óbito fetal, óbito neonatal, prematuridade, ou síndromes de sífilis congênita ${ }^{1,2}$. O tratamento com penicilina benzatina é altamente eficaz, proporcionando uma redução da transmissão vertical em até $97 \% \%^{2}$. Na ausência do tratamento, a transmissão vertical da sífilis é elevada, podendo alcançar valores próximos a $100 \%$ nas formas recentes da doença $a^{2,3}$.

Historicamente, o número de novos casos de sífilis sofreu um importante declínio a partir de 1940, com a introdução da penicilina no cenário médicocientífico $^{4}$. No Brasil, a notificação de sífilis congênita tornou-se compulsória em todo território nacional por meio da Portaria $n^{\circ} 542$, de 22 de dezembro de $1986^{5}$. Apesar de haver a redução de $55,94 \%$ donúmero demortes porsífilis em 2010 quando comparada a $1990^{6}$, os boletins epidemiológicos evidenciaram o aumento do número de casos de sífilis

Afiliação dos autores: 'Laboratório de Insetos Vetores, Universidade de Vassouras, Vassouras, RJ, Brasil.

${ }^{2}$ Pró-Reitoria de Ciências da Saúde, Universidade de Vassouras, Vassouras, RJ, Brasil.

${ }^{3}$ Mestrado Profissional em Ciências Ambientais, Universidade de Vassouras, Vassouras, RJ, Brasil.

${ }^{4}$ Mestrado Profissional em Ciências Aplicadas em Saúde, Universidade de Vassouras, Vassouras, RJ, Brasil

${ }_{5}^{5}$ aboratório de Entomologia Médica e Forense, Instituto Oswaldo Cruz/FIOCRUZ, Brasil.

* Email para correspondencia: adriano4353@hotmail.com 
congênita no Brasil nos últimos anos ${ }^{5,7,8,9}$. Junto a isso, ocorreu, desde 2014, a falta de penicilina benzatina por desabastecimento por falta de matéria prima para a sua produção ${ }^{9}$, uma vez que esse é o medicamento de primeira escolha para o controle da transmissão vertical ${ }^{1,2,4,9}$. O diagnóstico e tratamento adequado das gestantes com sífilis é muito importante para prevenira doença, uma vez que o tratamento é altamente eficaz, proporcionando a redução da transmissão vertical em até $97 \%^{2}$, e na ausência do tratamento, a transmissão vertical da sífilis é elevada, podendo alcançar valores próximos a $100 \%$ nas formas recentes da doença ${ }^{2}$.

$\mathrm{O}$ número de casos notificados depende não somente da capacidade de intervenção dos serviços de saúde como da atenção básica e atenção a gestante para reduzir a transmissão vertical, diagnosticando e tratando adequadamente as gestantes e seus parceiros, mas também da capacidade de identificação e notificação dos casos de sífilis congênita por parte da equipe médica ${ }^{5}$. Dessa forma, um número baixo de casos de sífilis congênita não indica necessariamente um bom programa de controle da transmissão vertical, uma vez que há a hipótese fatídica de estar havendo um falso negativo, quando casos de sífilis congênita podem estar ocorrendo, sem serem reconhecidos e/ou notificados. Por outro lado, um número elevado indica falhas no processo assistencial, com oportunidades perdidas de intervenção ${ }^{5}$.

Este estudo teve como objetivo realizar um levantamento epidemiológico da incidência de sífilis congênita no município de Vassouras, no estado do Rio de Janeiro e no Brasil. Os dados resultantes deste estudo poderão auxiliar os gestores em saúde quanto a situação epidemiológica do município e possibilitar ações preventivas e campanhas de conscientização à população.

\section{Material e Métodos}

O levantamento bibliográfico foi realizado nas plataformas Medline e Scielo, no período de 2006 a 2017 sobre a incidência de sífilis congênita. A busca de dados foi realizada a partir dos informes epidemiológicos do município de Vassouras, RJ, do estado do Rio de Janeiro e do Brasil, sobre sífilis, epidemiologia nacional e sífilis congênita nos últimos 15 anos, utilizando como descritores: boletim epidemiológico - sífilis; informe epidemiológico - sífilis materna e congênita.

\section{Resultados}

Os resultados anuais possibilitaram avaliar a evolução dos números de casos de sífilis ao longo dos últimos 15 anos. A taxa de incidência de sífilis congênita (por mil nascidos vivos) (Figura 1) no Brasil e no Estado do Rio de Janeiro cresceu ao longo dos últimos anos. No Brasil, a incidência passou de 1,1 casos de sífilis congênita em 2001 para 6,5 casos no ano de 2015. No período de 2001 até 2010 houve um crescimento de 1,1 para 2,4 casos em 10 anos. Entretanto, no período de 2010 até 2015, os casos aumentaram de 2,4 para 6,5 em apenas 6 (seis) anos. No estado do Rio de Janeiro a incidência ficou parcialmente estável variando de 6 a 7 casos no período de 2001 até 2010 . De 2010 até 2015 a mesma aumentou de 7 para 13,6 casos. Em contrapartida, o município de Vassouras revelou uma taxa de incidência de sífilis congênita de 3 (2001); 2 (2003); 2 (2005); 2,3 (2012) e de 4,4

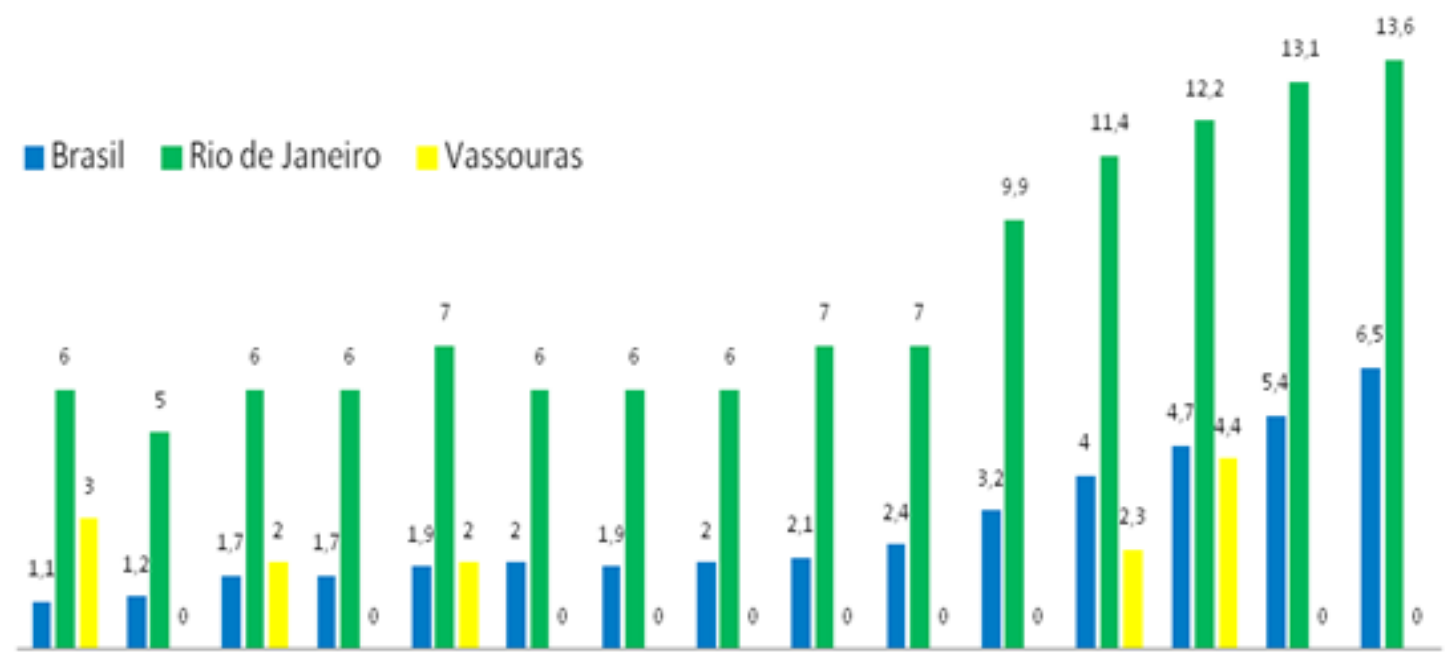

200120022003200420052006200720082009201020112012201320142015

Figura 1: Taxa de incidência de sífilis congênita (por mil nascidos vivos), de acordo com os dados dos informes epidemiológicos ${ }^{3,5,7,8,9}$. 
(2013) casos. Estas taxas são inferiores as observadas aos índices do Brasil e do Rio de Janeiro, apresentando inclusive incidência zero em dez anos dos quinze anos analisados.

\section{Discussão}

Os resultados são inquietantes, pois mesmo sendo a sífilis uma doença de fácil diagnóstico e tratamento, nos últimos quinze anos apresentou um aumento da taxa de incidência no Brasil e no estado do Rio de Janeiro, distanciando, cada vez mais das metas estabelecidas pelo Ministério da Saúde e pela Organização Pan-americana de Saúde, sendo que para a OPS a meta ideal é uma taxa inferior a 0,5 casos para cada mil nascidos vivos ${ }^{3}$.

A situação do estado do Rio de Janeiro foi bastante difícil durante os anos estudados, pois a taxa de incidência de sífilis congênita foi maior do que o dobro da média nacional, quando comparados aos outros estados da federação. O estado do Rio de Janeiro foi o que apresentou o maior aumento, no período estudado ${ }^{7,8,9}$, com acentuado crescimento a partir de 2011. Neste mesmo período foi implantado o Programa Rede Cegonha visando melhorar a atenção básica às gestantes no pré-natal com elevação na taxa de detecção de sífilis em gestantes decorrente da melhoria do diagnostico precoce e do aperfeiçoamento da vigilância epidemiológica ${ }^{7}$.

No caso do município de Vassouras, os resultados quanto à taxa de incidência de sífilis congênita, mostraram-se inferiores aos municípios de Paracambi ${ }^{3}$, Três Rios, ao estado do Rio de Janeiro e da média nacional. Este resultado está em consonância com a meta proposta pelo Ministério da Saúde em dez dos quinze anos analisados ${ }^{3}$.

Toda a preocupação é necessária, pois uma baixa incidência de sífilis congênita não significa uma boa atenção à saúde da gestante, mas pode sugerir casos de subnotificação ${ }^{3}$. No entanto, a alta taxa de incidência da doença, pode revelar muitas vezes, a deficiência do atendimento adequado à gestante. Apesar da penicilina benzatina ser o medicamento de primeira escolha para o tratamento e prevenção da sífilis congênita ${ }^{1,2,4}$, não foi realizado neste estudo a associação entre o aumento dos casos de sífilis congênita e à escassez do medicamento. pública no Brasil, pois é uma doença infectocontagiosa, cujo diagnóstico e tratamento são de fácil acesso e baixo custo. É possível que a elevada taxa de sífilis congênita possa ser um marcador de qualidade da atenção básica a saúde, revelando a situação de carência que se encontra a nação brasileira e o estado do Rio de Janeiro quanto aos seus serviços de Atenção Básica a Saúde da Mulher.

\section{Referências}

1-Edward W. Sífilis. In: Goldman L, Cecil AD. Tratado de Medicina Interna. 24. ed. Rio de Janeiro: Elsevier; 2015. p. 2221-29.

2-Kasper DL, Fauci AS, Hauser SL, Longo DL, Jamerson JL, Loscalzo J, et al. Sífilis. In: Medicina interna de Harrison. 18. ed. Porto Alegre: AMGH; 2013. p. $1380-88$.

3- Governo do estado do Rio de Janeiro. Secretaria de estado de saúde. Informe epidemiológico Sífilis materna e congênita. Rio de Janeiro (RJ); 2016.

4- Avelleira JCR, Bottino G. Sífilis: Diagnóstico, tratamento e controle. An Bras Dermatol. 2006; 81 (2): 111-26.< http://www.scielo.br/pdf/abd/v81n2/ v81n02a02.pdf>

5-Araújo CL, Shmizu HE, Sousa AIA, Hamann EM. Incidência da Sífilis Congênita no Brasil e sua relação com a Estratégia de Saúde da Família. Rev Saúde Pública. 2012; 46 (3): 479-86. <http://www.scielo.br/pdf/rsp/ v46n3/3477.pdf>

6- Lozano, R. Global and Regional Mortality from 235 causes of death for 20 age groups in 1990 and 2010: a systematic analysis for the Global Burden of Disease Study 2010. Lancet. 2012; 380: 2095-128. <https://www.ncbi.nlm. nih.gov/pubmed/23245604>

7- Ministério da Saúde. Secretaria de Vigilância em Saúde. Boletim Epidemiológico - Sífilis. Brasília, DF; 2015. <http://www.aids.gov.br/es/ node $/ 88>$.

8- Ministério da Saúde. Secretaria de Vigilância em Saúde. Boletim Epidemiológico - Sífilis. Brasília, DF; 2012. < http://www.aids.gov.br/pt-br/ node $/ 89>$.

9- Ministério da Saúde. Secretaria de Vigilância em Saúde. Boletim Epidemiológico - Sífilis. Brasília, DF; 2016. http://www.aids.gov.br/pt-br/ pub/2016/boletim-epidemiologico-de-sifilis-2016.

\section{Conclusão}

Este estudo epidemiológico demonstrou que a sífilis congênita ainda é um sério problema de saúde 